“( 2014 IEEE. Personal use of this material is permitted. Permission from IEEE must be obtained for all other uses, in any current or future media, including

reprinting/republishing this material for advertising or promotional purposes, creating new collective works, for resale or redistribution to servers or lists, or reuse of any copyrighted component of this work in other works." 


\section{Multimode Plasmon Resonances on Double- and Triple-decker Stacks of Silver Nanotriangles}

\author{
N. Shahcheraghi, A.Dowd, A.McDonagh, M.B. Cortie \\ Institute for Nanoscale Technology \\ University of Technology Sydney \\ NSW, Australia \\ michael.cortie@uts.edu.au
}

\begin{abstract}
Nanotriangles of silver may be readily synthesized by wet chemical techniques and exhibit a strong localized surface plasmon resonance with light. Here we examine the complex resonances of nanotriangles in double- and triple-decker sandwich configurations.
\end{abstract}

Keywords—nano-triangles; plasmon resonance; multimodal

\section{INTRODUCTION}

Silver is an attractive material for making discrete, nanoscale plasmonic devices because, unlike gold, it exhibits low loss over most of the visible spectrum. This is the result of it having a relatively low value of $\varepsilon_{2}$, the imaginary part of dielectric function $\varepsilon=\varepsilon_{1}+i . \varepsilon_{2}$. Therefore, localized surface plasmon resonances developed on appropriately designed single crystal nanostructures of silver can be very intense. Importantly, this permits the observation of multimodal or higher-order resonances [1] that are normally obscured on less optimum nanostructures [2]. Another attraction of working with silver nanoparticles is that they can be induced to selfassemble into interesting configurations, such as dimers or, of relevance here, double- and triple-decker stacks. The stacks were selected for detailed study as they have evidently not been investigated before, can be produced by self-assembly, and offer the prospect of being able to host complex and interesting multimode resonances.

\section{EXPERIMENTAL}

Samples of silver nanotriangles were prepared by reacting $\mathrm{AgNO}_{3}$ with $\mathrm{NaBH}_{4}$ to produce nanoparticle nuclei, and then growing these in a solution containing trisodium citrate, $\mathrm{H}_{2} \mathrm{O}_{2}$ and polyvinylpyrrolidone (the latter as a protective surfactant). This is a modified version of a technique previously described by Tsuji et al. [3], The optical properties of these nanoparticles were examined both in colloidal suspension and after deposition of glass or silicon substrates, using appropriate spectrometers. The properties of a variety of configurations of nanotriangles were investigated using the Discrete Dipole Approximation as implemented in the program DDSCAT [4]. The electric fields and multimodal nature of the resonances were visualized with software written by one of the authors (M.B.C.).

\author{
V. Keast \\ School of Mathematical and Physical Sciences \\ University of Newcastle \\ NSW, Australia
}

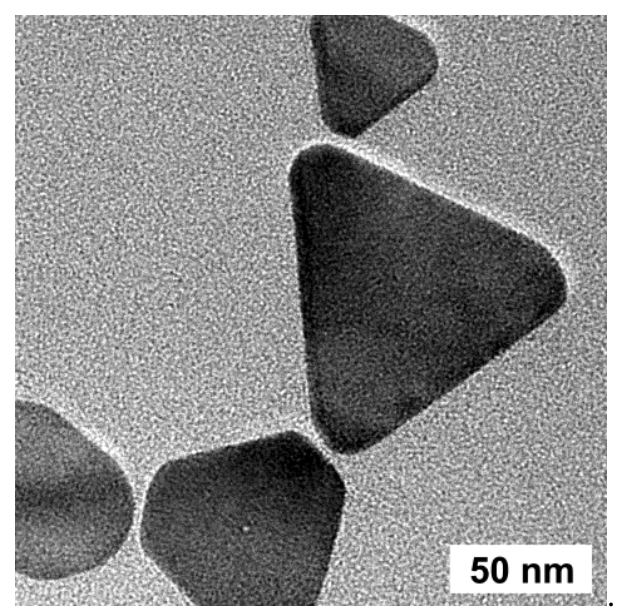

Fig.1. Transmission electron microscope image of experimentally produced silver nanotriangles. Both normal and 'truncated' versions of the nanoparticle are evident.

\section{RESULTS}

Silver nanotriangles of a range of shapes were produced. Examination in the transmission electron microscope, Fig. 1, showed that individual particles were single crystals, with the flat faces of the particles being $\{111\}$ planes, as attested by the six-fold atomic symmetry in high resolution TEM images and their electron diffraction patterns. This is in agreement with the literature [5]. In general the edge lengths of the triangles were in the range $100 \pm 20 \mathrm{~nm}$, but some shapes were truncated into a hexagonal geometry. The facets of these shapes were of the order of $60 \pm 20 \mathrm{~nm}$ long. The thickness of the shapes was determined to be of the order of 20 to $30 \mathrm{~nm}$ on the strength of some of the particles being observed edgeon. A range of reactant ratios and concentrations were explored and it was found that successful synthesis depended on having very fresh $\mathrm{NaBH}_{4}$. Use of older samples of the reductant was associated with the production of large spheres or other undesirable shapes.

The UV-visible extinction spectrum of the colloid revealed a sharp absorption peak at about $830 \mathrm{~nm}$, Fig. 2(a), indicating a predominance of particles with a relatively high aspect ratio. This generated a blue color to the eye, Fig. 2(b). However, the reflectance spectrum of samples that had been 

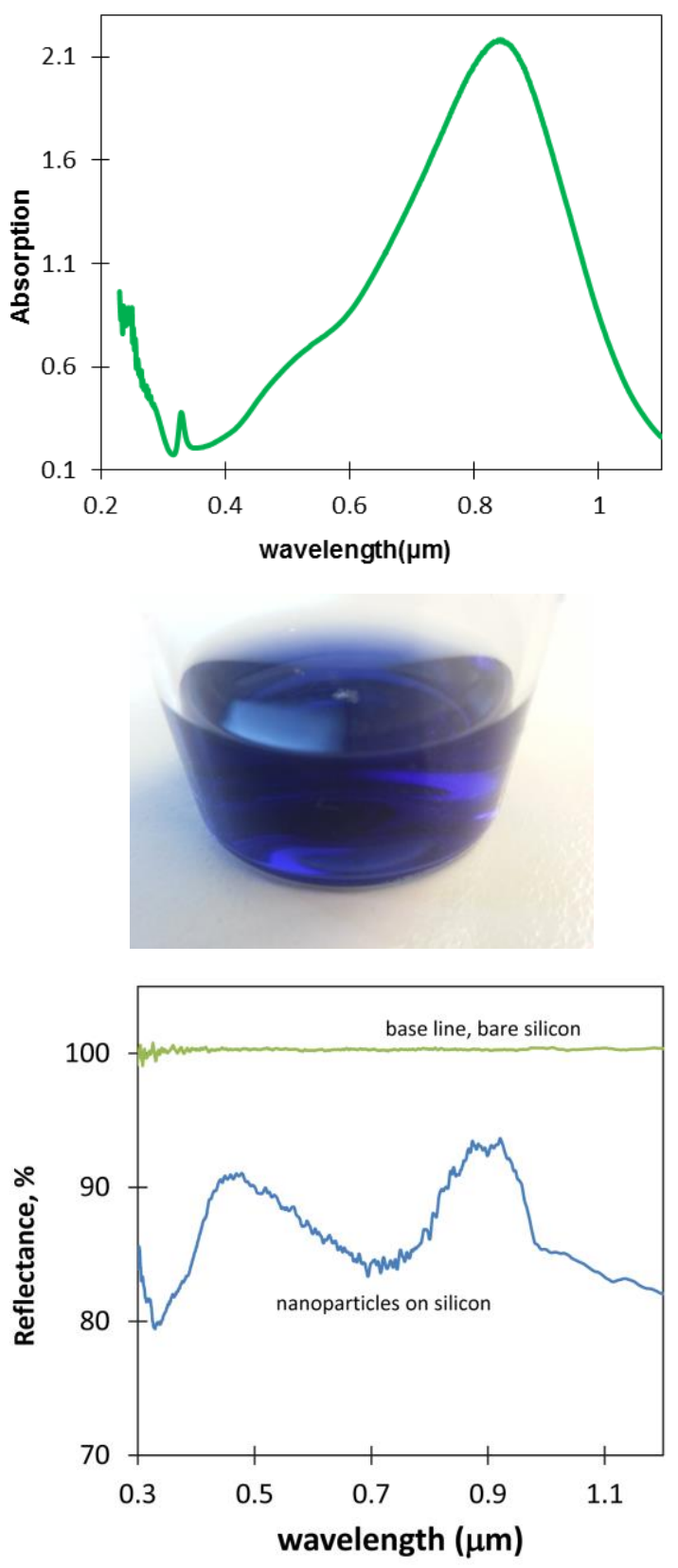

Fig. 2. (a) UV-visible extinction spectrum of a typical colloidal suspension of Ag nanoparticles. (b) The broad absorption peak in the upper visible and near infrared causes the sample to display a deep blue color. (c) Reflectance spectrum of a dispersion of particles that had been dried out on a silicon substrate. The blue-shifting of the main dipolar resonance peak to $0.7 \mu \mathrm{m}$ is evident.

dried on silicon or glass substrates showed a considerably blue-shifted absorption peak at about $700 \mathrm{~nm}$, Fig. 2(c). This is at too long a wavelength to be due to a dipole resonance in the short transverse direction of the particle. A possible explanation for the blue-shift was that the triangles placed on the substrates had assembled into stacks on drying. This was confirmed by examination of scanning electron microscope (SEM) images.
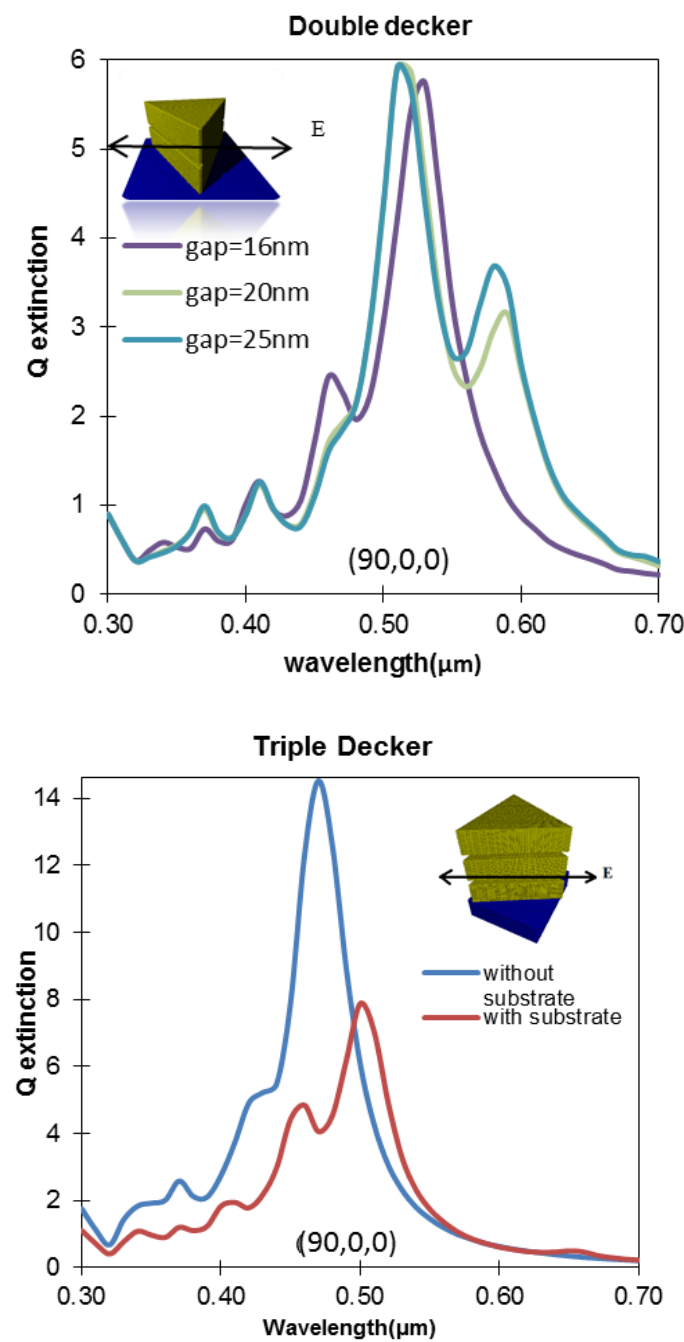

Fig. 3. Calculated extinction efficiencies of $80 \times 80 \times 80 \times 20 \mathrm{~nm}$ silver nanotriangles in double- and triple-decker configurations. The substrate, where present, is glass.

A prototypical triangular shape of $80 \times 80 \times 80 \times 20 \mathrm{~nm}$ was selected for the purpose of examining the resonances of the triangles by simulation. DDA calculations of double- or tripledecker stacks of such triangles predicted absorption peaks at about $500 \mathrm{~nm}$, Fig.3. The nature of the resonances responsible for the various extinction peaks was ascertained using a combination of computer animations and plots showing magnitude, direction or phase of the fields. The oscillation excited at, for example, $500 \mathrm{~nm}$ when a double-decker stack is illuminated with the direction of polarization parallel to the flat faces of the nanotriangles is shown in Fig.4. It is clear that a dipole resonance is being excited in this case, although, because there are two triangles, there is a relatively complex symmetry at the apices of the shapes, and the wavelength at which the dipole resonance occurs has been considerably blueshifted relative to that of an isolated triangle, Fig. 5. The electric field on the plane equidistant between the two triangles is close to zero because the surface charges on the faces are of the same polarity. 

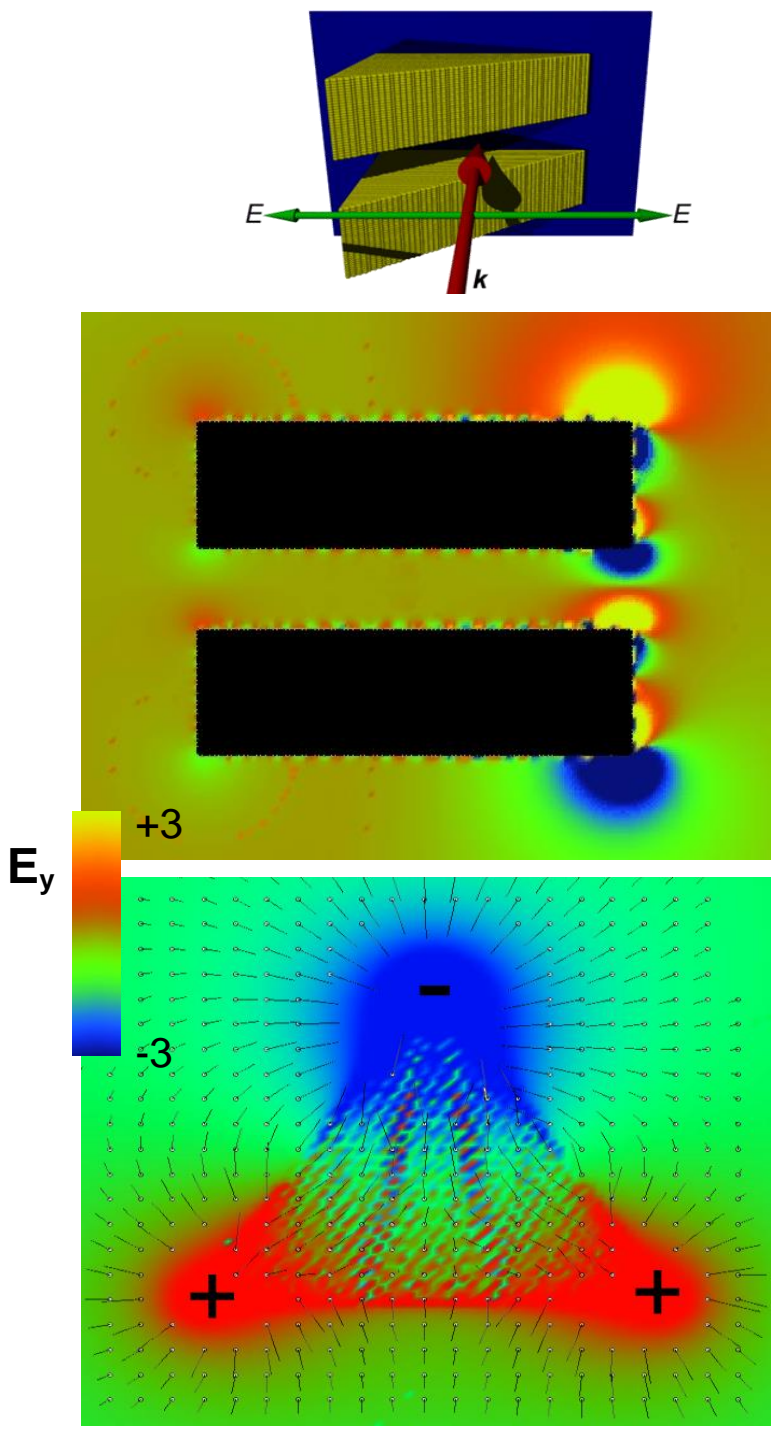

Fig. 4. Calculated electric field distribution in a double-decker stack illuminated at $500 \mathrm{~nm}$ (a) Geometric configuration showing direction of light and placement of cross-section of electric field, (b) cross-section of electric field showing y-component ( $y$ is normal to $\mathbf{E}$ and $\mathbf{k})$ ). (c) ycomponent of electric field calculated on a plane $1 \mathrm{~nm}$ above the top face of upper triangle.

Multimode resonances of even more complex symmetry were identified at shorter wavelengths, Fig. 6. At $422 \mathrm{~nm}$ there

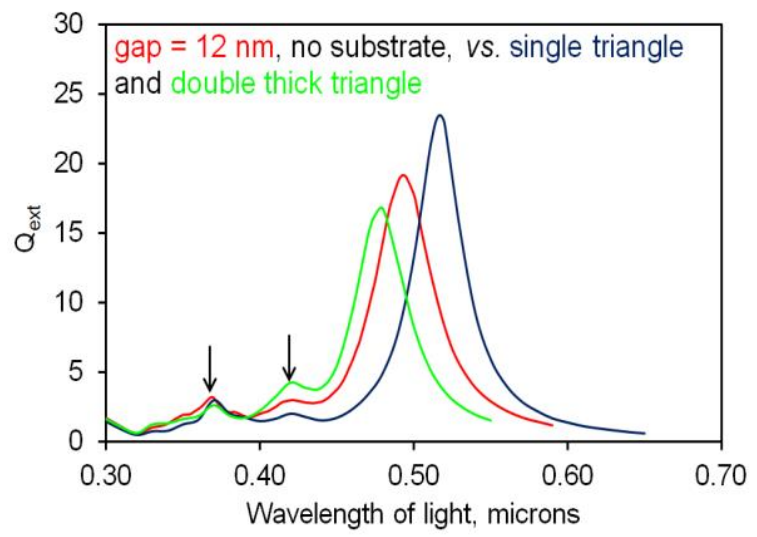

Fig. 5. Calculated extinction efficiency of an isolated Ag nanotriangle $(80 \times 80 \times 80 \times 20 \mathrm{~nm})$ versus a double-decker sandwich with a $12 \mathrm{~nm}$ gap and a single Ag nanotriangle of $80 \times 40 \mathrm{~nm}$ (equivalent to a gap-less double-decker sandwich). The positions of the $369 \mathrm{~nm}$ (decapolar) and $422 \mathrm{~nm}$ (octapolar) resonances are indicated.

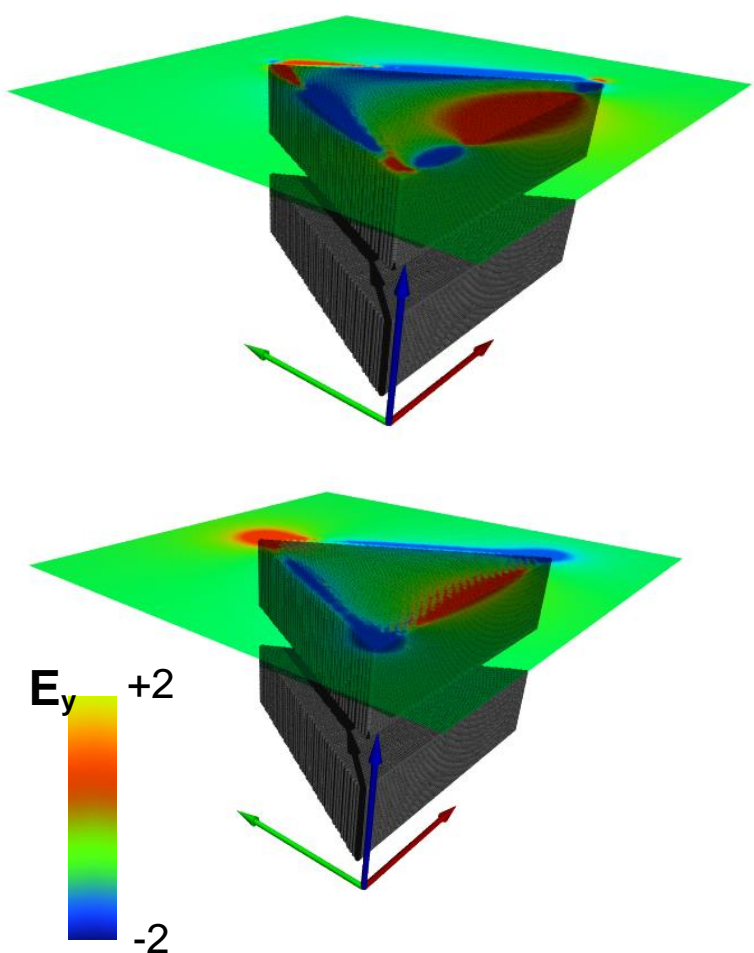

Fig. 6. Complex multipolar plasmon resonances of a double-decker pair of $\mathrm{Ag}$ nanotriangles of $80 \mathrm{~nm} \times 20 \mathrm{~nm}$, with a $12 \mathrm{~nm}$ gap, when illuminated at (a) $369 \mathrm{~nm}$, (b) $422 \mathrm{~nm}$. $\mathbf{E}$ is in direction of blue arrow, $\mathbf{k}$ in direction of red arrow.

is a resonance that has an octapolar nature whereas at $369 \mathrm{~nm}$ a decapolar resonance is excited. Examination of the calculated extinction spectra as a function of the gap distance between the triangles shows that the wavelengths at which these multipolar resonances occur is scarcely affected by the gap distance. In contrast, the wavelength at which the dipole resonance peaks is quite sensitive to the gap distance. The explanation for this is that the multipolar resonances have short-range spatial features and therefore are hardly influenced by the surface charge on the neighboring triangle. In contrast, the dipole resonances generate an electric field distribution that extends much further out into free space, so that the presence of the neighboring triangle has a strong influence.

The simulations also revealed another interesting aspect of

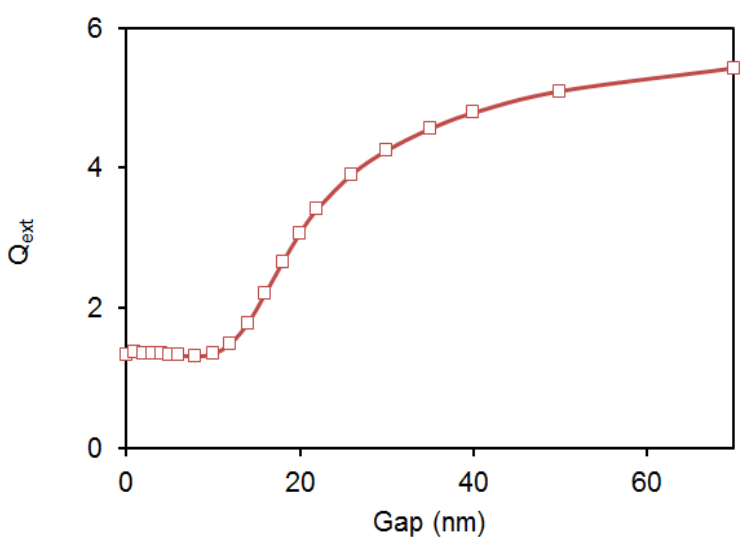

Fig. 7. Calculated extinction efficiencies at $580 \mathrm{~nm}$ for a series of double-decker $\mathrm{Ag}$ nanotriangles on glass, as a function of the gap between them. 
the dipole-dipole coupling between upper and lower triangles. When the sandwich is on a glass substrate (Fig. 3 for example), the degeneracy of the dipole resonance on the two triangles will be broken if the gap between them is greater than about $10 \mathrm{~nm}$. In these cases there are two distinct dipole resonances, with that on the lower triangle red-shifted by about $50 \mathrm{~nm}$ due to the effect of the glass substrate. However, when the gap between the triangles is smaller than about 10 $\mathrm{nm}$ the electric fields across the gap are so tightly coupled that the two triangles behave as one, with their respective resonances hybridized to the same frequency. The intensity of the resonance on the lower triangle is shown in Fig. 7 using the value of $Q_{\text {ext }}$ at $580 \mathrm{~nm}$ as a proxy.

\section{CONCLUSIONS}

Silver nanotriangles may be prepared by a wet chemical technique but, in our experience, the product consists of a diverse range of shapes. A prototypical nano-triangle of $80 \times 80 \times 80 \times 20 \mathrm{~nm}$ was selected here for further study and examined in double-and triple-decker configurations. The primary effect of including a glass substrate in the simulations is to red-shift the dipole plasmon resonances, particularly that of the triangle adjacent to the glass. As a result, the dipole resonance of the structure splits into two components of slightly differing energy. The degree of splitting depends on the gap between the two triangles. For gaps of less than $10 \mathrm{~nm}$ the plasmons of the two triangles are fully hybridized, and the splitting is minimal, however the as the gap size increases beyond $10 \mathrm{~nm}$, the plasmons on the two triangles de-couple and their characteristic resonant frequencies separate.

These shapes also display two distinct multimodal resonances at higher energies. Due to their short-range spatial complexity these resonances are not much affected by the magnitude of the gap and, for the same reason, have scarcely any effect on the far-field extinction. They may, nevertheless, be technologically interesting on account of the complex local fields that they generate.

\section{ACKNOWLEDGMENT}

This work has been supported by the Australian Research Council under DP 120102545. We thank Dr M. Arnold of UTS for useful discussions.

\section{REFERENCES}

[1] M. B. Cortie, F. Liu, M. D. Arnold and Y. Niidome, "Multimode Resonances in Silver Nanocuboids". Langmuir, vol.28, pp.9103-9112, 2012.

[2] M. G. Blaber, M. D. Arnold and M. J. Ford, "Optical properties of intermetallic compounds from first principles calculations: a search for the ideal plasmonic material". Journal of Physics: Condensed Matter, vol.21, pp.144211, 2009.

[3] M. Tsuji, S. Gomi, Y. Maeda, M. Matsunaga, S. Hikino, K. Uto, T. Tsuji and H. Kawazumi, "Rapid Transformation from Spherical Nanoparticles, Nanorods, Cubes, or Bipyramids to Triangular Prisms of Silver with PVP, Citrate, and H2O2". Langmuir, vol.28, pp.8845-8861, 2012.

[4] B. T. Draine and P. J. Flatau, "Discrete-dipole approximation for periodic targets: theory and tests". J. Opt. Soc. Am. A, vol.25, pp.2693-2703, 2008.

[5] L. J. Sherry, R. Jin, C. A. Mirkin, G. C. Schatz and R. P. Van Duyne, "Localized Surface Plasmon Resonance Spectroscopy of Single Silver Triangular Nanoprisms". Nano Letters, vol.6, pp.2060-2065, 2006. 